\title{
The Central Vein Sign in Radiologically Isolated Syndrome
}

\author{
(D)S. Suthiphosuwan, (DP. Sati, (D) M. Guenette, (D) X. Montalban, (DD.S. Reich, (D)A. Bharatha, and (D). Oh
}

\begin{abstract}
BACKGROUND AND PURPOSE: Radiologically isolated syndrome describes asymptomatic individuals with incidental radiologic abnormalities suggestive of multiple sclerosis. Recent studies have demonstrated that $>40 \%$ of white matter lesions in MS (and often substantially more) have visible central veins on MR imaging. This "central vein sign" reflects perivenous inflammatory demyelination and can assist in differentiating MS from other white matter disorders. We therefore hypothesized that $>40 \%$ of white matter lesions in cases of radiologically isolated syndrome would show the central vein sign.
\end{abstract}

MATERIALS AND METHODS: We recruited 20 participants diagnosed with radiologically isolated syndrome after evaluation by a neurologist. We performed 3T MR imaging of the brain and cervical spinal cord. White matter lesions were analyzed for the central vein sign.

RESULTS: Of 391 total white matter lesions, 292 (75\%) demonstrated the central vein sign (central vein sign+). The median proportion of central vein sign + lesions per case was $87 \%$ (range, $29 \%-100 \%$ ). When the " $40 \%$ rule" that has been proposed to distinguish MS from other disorders was applied, of 20 participants, 18 cases of radiologically isolated syndrome $(90 \%)$ had $\geq 40 \%$ central vein sign + lesions (range, $55 \%-100 \%)$. Two participants (10\%) had $<40 \%$ central vein sign + lesions ( $29 \%$ and $31 \%)$. When the simpler "rule of 6 " was applied, 19 participants (95\%) met these criteria. In multivariable models, the number of spinal cord and infratentorial lesions was associated with a higher proportion of central vein sign + lesions $(P=.002 ; P=.06$, respectively).

CONCLUSIONS: Most cases of radiologically isolated syndrome had a high proportion of central vein sign + lesions, suggesting that lesions in these individuals reflect perivenous inflammatory demyelination. Moreover, we found correlations between the proportion of central vein sign + lesions and spinal cord lesions, a known risk factor for radiologically isolated syndrome progressing to MS. These findings raise the possibility, testable prospectively, that the central vein sign may have prognostic value in distinguishing patients with radiologically isolated syndrome at risk of developing clinical MS from those with white matter lesions of other etiologies.

ABBREVIATIONS: CVS = central vein sign; RIS = radiologically isolated syndrome

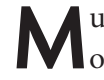
ultiple sclerosis is an inflammatory demyelinating disease of the central nervous system characterized by relapsing or progressive neurologic symptoms and focal white matter lesions. ${ }^{1}$ Histopathologic studies have shown that these WM lesions, when

Received January 28, 2019; accepted after revision March 25.

From the Division of Neuroradiology (S.S., A.B.), Division of Neurology (S.S., M.G., X.M., J.O.), Department of Medicine, and Division of Neurosurgery (A.B.), Department of Surgery, St. Michael's Hospital, University of Toronto, Toronto, Ontario, Canada; Translational Neuroradiology Section (P.S., D.S.R.), National Institute of Neurological Disorders and Stroke, National Institutes of Health, Bethesda, Maryland; and Department of Neurology (D.S.R., J.O.), Johns Hopkins University, Baltimore, Maryland.

This work was supported by the MS Society of Canada and the St. Michael's Hospital Foundation Love/Barford Endowment.

The funders had no role in the design and conduct of the study; collection, management, analysis, and interpretation of the data; preparation, review, or approval of the manuscripts; and the decision to submit the manuscript for publication.

Paper previously presented at: American Society of Neuroradiology Annual Meeting and the Foundation of the ASNR Symposium, June 2-7, 2018, Vancouver, British Columbia, Canada (abstract No. O-70). they first form, arise from inflammatory infiltrates around small veins, or "perivascular cuffs." ${ }^{2}$ On conventional MR imaging, MS lesions typically manifest as focal areas of high signal on T2weighted images of the brain and spinal cord. However, focal T2-hyperintense WM lesions can be seen in a variety of settings, including normal aging, migraines, microangiopathy, and CNS vasculitis. Misinterpretation of such lesions is the most common cause of incorrect MS diagnosis, ${ }^{3}$ and partly to address this issue, one can only apply the current MS diagnostic criteria in the setting of typical clinically isolated syndrome. ${ }^{4-6}$ Diagnosing MS when such a syndrome is not present is therefore challenging.

Recent studies using high-resolution, susceptibility-based MR imaging sequences have demonstrated an identifiable vein inside

Please address correspondence to Jiwon Oh, MD, FRCPC, PhD, 30 Bond St, Shuter 3-019, Toronto, ON, M4K 1Z1 Canada; e-mail: ohjiw@smh.ca

http://dx.doi.org/10.3174/ajnr.A6045 
WM lesions, called the "central vein sign" (CVS)., ${ }^{3,-21}$ On these scans, the CVS is typically visible as a hypointense line that is centered within the lesion; the hypointensity reflects the presence of a paramagnetic substance, either deoxyhemoglobin or, in some circumstances, a gadolinium-based contrast agent. ${ }^{9,11,12}$ Previous studies assessing the CVS in MS have reported that a high frequency of WM lesions in MS demonstrate the CVS compared with WM lesions seen in microangiopathic disease, migraines, CNS inflammatory vasculopathies, and neuromyelitis optica spectrum disorders. ${ }^{3,8,10,13-17,19-23}$ On the basis of these studies, a cutoff threshold of $40 \%$ (in which $\geq 40 \%$ of WM lesions demonstrate the CVS) has been proposed to distinguish MS from non-MS disease. ${ }^{8}$ More recent studies have proposed more simplified criteria, such as the "rule of 6 ," whereby if $>6$ WM lesions demonstrate the CVS, MS can be distinguished from other WM disorders. ${ }^{10,21,23}$

Radiologically isolated syndrome (RIS) describes asymptomatic individuals or those with neurologic symptoms that are not typical of MS, whose MR imaging scans show abnormalities typical of those seen in clinical MS. ${ }^{24}$ Individuals with RIS have an increased risk of eventually developing clinical MS, but long-term prospective studies are lacking. The largest, multicenter, retrospective study has shown that $34 \%$ of patients with RIS eventually manifest clinically definite MS during 4.4 years. ${ }^{25}$ Factors that increase the risk of conversion to MS include the following: male sex, younger age (younger than 37 years of age), and the presence of $\geq 1$ spinal cord lesion on MR imaging. ${ }^{25,26}$ Another study demonstrated that among individuals with RIS who develop MS, $11.7 \%$ develop primary-progressive MS, and these individuals are more likely to be older and male and have spinal cord lesions. ${ }^{27}$ These data suggest that at least a portion of patients with RIS have a subclinical form of MS; of these, many may never develop clinical MS.

Currently, there is no consensus on the optimal clinical management of RIS because the natural history remains unclear and there are no evidence-based treatment or management guidelines. Moreover, the current diagnostic criteria for RIS ${ }^{24}$ likely result in a heterogeneous group of participants being designated as such, including those who are at risk of developing MS and those who have a minimal risk. This result is likely because the diagnostic criteria for RIS are based entirely on lesion number and distribution (Barkhof ${ }^{28}$ criteria for dissemination in space), criteria that were originally intended to be applied to individuals with symptomatology clinically suggestive of demyelinating disease. As a result, the current RIS criteria ${ }^{24}$ have limited ability to reflect underlying subclinical demyelinating pathology, which represents individuals at high risk of developing MS. ${ }^{29}$ Accordingly, assessing the CVS in WM lesions of RIS may be helpful to better evaluate the underlying pathologic processes of this entity and determine which individuals are at risk of eventually developing clinical MS. ${ }^{30}$ To date, the presence of the CVS has not been evaluated in RIS, to our knowledge. Therefore, the objective of this study was to determine whether adults with RIS have similar proportions of lesions positive for CVS (CVS+ lesions) compared with those previously reported in clinical MS.

\section{MATERIALS AND METHODS Standard Protocol Approvals, Registrations, and Patient Consent}

This study was approved by the institutional review board of St. Michael's Hospital. All participants provided written informed consent.

\section{Participants}

Twenty adults diagnosed with RIS by a neurologist at the St. Michael's Hospital MS Clinic were recruited for prospective clinical and MR imaging evaluation between July 2017 and December 2017. Because RIS is a rare entity ${ }^{31}$ and the central vein sign has not yet been evaluated in RIS, we were not able to perform sample size calculations of power analyses. Inclusion criteria were 18 years of age and older and meeting previously published clinical and MR imaging criteria for RIS, ${ }^{24}$ which include the presence of asymptomatic WM abnormalities that are ovoid, wellcircumscribed, $\geq 3 \mathrm{~mm}$ in maximal diameter, and fulfilling 3 of 4 Barkhof criteria $^{28}$ for dissemination in space (lesions in the periventricular, juxtacortical, infratentorial, or spinal cord regions). Brain MRIs were reviewed by experienced neuroradiologists (S.S. and A.B.) and a neurologist (J.O.) to confirm inclusion criteria. Individuals who had vascular risk factors and a history of substance abuse or toxic exposure were excluded. ${ }^{24}$

\section{Clinical Assessment}

Within 30 days of MR imaging, study participants underwent an examination by a neurologist. None of the participants experienced a clinical relapse or neurologic symptoms suggestive of MS between recruitment and the study visit.

\section{MR Imaging}

MR imaging was performed on a 3T scanner (Magnetom Skyra; Siemens, Erlangen, Germany) with a 20-channel head-neck coil and a 16-channel spine-array coil.

\section{Brain MR Imaging}

The following parameters were used for brain sequences-3D T1weighted MPRAGE: TR/TE/flip angle $=1900 / 2.52 \mathrm{~ms} / 9^{\circ}$, number of averages $=1$, slice thickness $=1 \mathrm{~mm}$, in-plane resolution $=$ $1 \times 1 \mathrm{~mm}^{2}$, and number of slices $=176$; 3D T2-FLAIR: TR/TE/ $\mathrm{TI} /$ flip angle $=4800 / 353 / 1800 \mathrm{~ms} / 120^{\circ}$, number of averages $=1$, slice thickness $=1 \mathrm{~mm}$, in-plane resolution $=1 \times 1 \mathrm{~mm}^{2}$, and number of slices $=176$; sagittal $3 \mathrm{D}$ T2 ${ }^{\star}$-weighted multishot echoplanar imaging: ${ }^{9} \mathrm{TR} / \mathrm{TE} /$ flip angle $=64 / 35 \mathrm{~ms} / 10^{\circ}$, number of averages $=1$, slice thickness $=0.65 \mathrm{~mm}$, in-plane resolution $=$ $0.65 \times 0.65 \mathrm{~mm}^{2}$, and number of slices $=265$.

\section{Cervical Spinal Cord MR Imaging}

The following parameters were used for cervical spinal cord sequences-sagittal 2D-T1-weighted phase-sensitive inversion recovery of the cervical spine: ${ }^{32} \mathrm{TR} / \mathrm{TE} / \mathrm{TI}=2400 / 9.4 / 400 \mathrm{~ms}$, number of averages $=2$, slice thickness $=3 \mathrm{~mm}$, in-plane resolution $=0.7 \times 0.7 \mathrm{~mm}^{2}$, and number of slices $=15$. A T2weighted sequence of the cervical spine was not performed. 


\section{Image Analysis}

Images were evaluated for the presence of the CVS by 3 reviewers: a neuroradiologist (S.S.), a medical physicist (P.S.), and a neurologist (J.O.). All reviewers completed training in CVS assessment according to the consensus criteria of the North America Imaging in Multiple Sclerosis Cooperative. ${ }^{11}$

\section{White Matter Lesion Evaluation}

3D-T1-MPRAGE and 3D-T2-FLAIR images were evaluated for the number, location, and size of WM lesions by a neuroradiologist (S.S.). WM lesion locations were classified into 4 typical locations of MS-related lesions in the brain, including cortical/juxtacortical (involving or touching the cortex), subcortical/deep, periventricular (touching 1 of the ventricles), and infratentorial. The size of the WM lesion was determined by assessing the largest diameter of the lesion across all planes. The WM lesions with hypointense signal lower than that on the cortex on T1-MPRAGE, so called T1 black holes, were recorded.

The Multi-Atlas Cortical Reconstruction Using Implicit Surface Evolution ${ }^{33}$ was used to acquire volumes of brain substructures, total lesion volume, and intracranial volume. The cerebral volume fraction was calculated by dividing total brain volume by intracranial volume.

\section{CVS Assessment}

$3 \mathrm{D}-\mathrm{T} 2^{\star}$ EPI magnitude images with multiplanar reconstruction were viewed in 3 orthogonal planes to assess the presence of the CVS using recently published guidelines. ${ }^{11}$ Specifically, identified central veins had to meet the following criteria: have a small apparent diameter $(<2 \mathrm{~mm})$; appear as a thin hypointense line or small hypointense dot; be visible in at least 2 perpendicular planes and appear as a thin hypointense line in 1 plane, run partially or entirely through the lesion; and be positioned approximately in the center of the WM lesion. WM lesions of $<3 \mathrm{~mm}$ in maximum diameter, confluent or contiguous lesions, lesions containing $>1$ vein, and poorly visible lesions were excluded from this analysis.

Two reviewers (S.S. and P.S.) independently evaluated brain MRIs and assessed each WM lesion that was designated as being positive for the CVS (CVS+), or not. If there was discordance between the 2 reviewers, a third reviewer (J.O.) assessed the lesion and a consensus was reached by discussion. The proportion of CVS+ WM lesions in each participant was expressed as a percentage of the total WM lesions in each participant. The CVS assessment was performed blinded to the presence of spinal cord lesions and clinical information.

We applied 2 previously published criteria using the CVS to distinguish MS from other WM disorders: the " $40 \%$ rule," whereby a diagnosis of MS is likely if an individual has $\geq 40 \%$ CVS + WM lesions; 9 and the "rule of 6," whereby a diagnosis of MS is likely if an individual has $\geq 6$ CVS + WM lesions. For the rule of 6 , if an individual has fewer than 6 CVS + WM lesions, but CVS + WM lesions outnumber non-CVS WM lesions, a diagnosis of MS can still be considered. If neither of these conditions is met, the diagnosis of MS is unlikely. ${ }^{10}$

\section{Cervical Spinal Cord Lesion Count}

Sagittal 2D phase-sensitive inversion recovery images of the cervical spine, ${ }^{32}$ which have been shown to have higher sensitivity for cervical spinal cord lesions over T2-based imaging, ${ }^{32,34}$ were used to assess for cervical spinal cord lesions. Phase-sensitive inversion recovery images were reviewed by 1 reviewer (S.S.), and the number of the cervical spinal cord lesions was evaluated, while the reviewer was blinded to the CVS analysis and clinical information.

\section{Statistical Analysis}

Statistical calculations were performed using SPSS (Version 23.0; IBM, Armonk, New York). Univariable and multivariable linear regression was used to identify independent variables associated with the proportion of CVS + WM lesions. Statistical significance was defined as $P<.05$.

\section{RESULTS}

\section{Clinical Characteristics of Participants with RIS}

This study included 20 adults with RIS. Fifteen participants (75\%) were women. and the mean age was 46.3 years (range, 25-62 years). None of the participants had any current or prior neurologic symptoms suggestive of demyelinating disease. The neurologic examination findings were normal in all except 4 subjects: Three had a mildly diminished vibration sensation in the distal lower limbs, and 1 subject had mild horizontal gaze-evoked nystagmus without diplopia. Headache was the most common reason $(9 / 20,45 \%)$ for the initial brain MR imaging being performed. CSF studies were available in 6 of 20 participants with RIS, and CSF-specific oligoclonal bands were detected in 5 participants. Alternative diagnoses were clinically excluded by the treating neurologist and included a negative systemic autoimmune screen in 18 of 20 participants. None of the subjects had undergone disease-modifying treatment used in MS. Table 1 summarizes clinical and MR imaging characteristics, and Table 2 lists the reasons for the initial brain MR imaging.

\section{MR Imaging Findings}

White Matter Lesions. A total of 997 WM lesions were identified. The median number of WM lesions per participant with RIS was 33 (range, 9-165). Two hundred six WM lesions (21\%) were T1 black holes. The number of WM lesions in specific brain regions was as follows: 193 cortical/juxtacortical lesions (19\%), 562 subcortical/deep WM lesions (56\%), 203 periventricular WM lesions (20\%), and 39 infratentorial lesions (4\%).

Nine participants with RIS (45\%) had lesions in the infratentorial brain. There were 16 participants with RIS (80\%) who had WM lesions involving the corpus callosum (49 WM lesions). T1 black hole lesions were found in 15 participants with RIS (75\%).

Automated brain segmentation ${ }^{33}$ was performed in 18 participants with RIS. The median brain volume was $1208 \mathrm{~cm}^{3}$ (range, $1066-1468 \mathrm{~cm}^{3}$ ), the median total brain lesion volume was 3.9 $\mathrm{cm}^{3}$ (range, $0.3-17.9 \mathrm{~cm}^{3}$ ), and the median cerebral volume fraction was 0.9 (range, $0.88-0.91$ ). Of note, the automated brain segmentation $^{34}$ failed in 2 cases (Table 1).

Cervical Spinal Cord Lesions. Thirteen participants (65\%) had at least 1 lesion in the cervical spinal cord. The median number of lesions within the cervical spinal cord was 1 (range, $0-4$ ) (Table 1).

Central Vein Sign Assessment. Of 997 WM lesions, 606 (61\%) were excluded from analysis according to previously published 
Table 1: Clinical and MRI characteristics of RIS participants

\begin{tabular}{|c|c|}
\hline \multicolumn{2}{|l|}{ Clinical Characteristics } \\
\hline Participants (No.) & 20 \\
\hline Age (mean) (SD) (yr) & $46(11)$ \\
\hline Female (No.) (\%) & $15(75 \%)$ \\
\hline No. of cases with positive oligoclonal band (No.) (\%) ${ }^{a}$ & $5(83 \%)$ \\
\hline \multicolumn{2}{|l|}{ MRI characteristics } \\
\hline \multicolumn{2}{|l|}{ Brain lesions } \\
\hline Total brain lesion count (No.) & 997 \\
\hline No. of brain lesions per case (median) (range) & $33(9-165)$ \\
\hline Total brain lesion volume (median) $\left(\right.$ range $\left(\mathrm{cm}^{3}\right)^{\mathrm{b}}$ & $3.9(0.3-17.9)$ \\
\hline No. of cases with T1 black hole lesions (No.) (\%) & $15(75 \%)$ \\
\hline No. of Tl black hole lesion count & $206(21 \%)$ \\
\hline No. of $\mathrm{Tl}$ black hole lesions per case (median) (range) & $3.5(0-43)$ \\
\hline No. of brain lesions included in the analysis (No.) (\%) & $391(39 \%)$ \\
\hline No. of brain lesions excluded from analysis (No.) (\%) & $606(61 \%)$ \\
\hline Brain volume (median) (range) $\left(\mathrm{cm}^{3}\right)^{\mathrm{b}}$ & $1208(1066-1468)$ \\
\hline Cerebral volume fraction (median) (range) ${ }^{\mathrm{b}}$ & $0.90(0.88-0.91)$ \\
\hline \multicolumn{2}{|l|}{ No. of assessed brain lesion/total brain lesion by region (\%) } \\
\hline Cortical/juxtacortical & $90 / 193(47 \%)$ \\
\hline Subcortical/deep & $228 / 562(40 \%)$ \\
\hline Periventricular & $60 / 203(30 \%)$ \\
\hline Infratentorial & $13 / 39(33 \%)$ \\
\hline \multicolumn{2}{|l|}{ Cervical spinal cord lesions } \\
\hline No. of cases with spinal cord lesions (No.) (\%) & $13(65 \%)$ \\
\hline Total spinal cord lesion count & 30 \\
\hline No. of spinal cord lesions per case (median) (range) & $1(0-4)$ \\
\hline
\end{tabular}

Table 2: Reasons for performing initial brain MRI

\begin{tabular}{lc}
\hline \multicolumn{1}{c}{ Reasons } & (No.) (\%) \\
\hline Headache & $9(45 \%)$ \\
Work-up for pituitary adenoma & $2(10 \%)$ \\
Transient paraphasic symptoms atypical for & $2(10 \%)$ \\
$\quad$ demyelinating disease & \\
Intermittent subjective cognitive symptoms & $1(5 \%)$ \\
Intermittent nocturnal tremor & $1(5 \%)$ \\
Pars planitis & $1(5 \%)$ \\
Sinusitis & $1(5 \%)$ \\
Back pain & $1(5 \%)$ \\
Dental pain & $1(5 \%)$ \\
Tinnitus & $1(5 \%)$ \\
\hline
\end{tabular}

CVS criteria, ${ }^{11}$ leaving 391 WM lesions (39\%) for analysis. Of these, 292 (75\%) were CVS+, whereas 99 (25\%) did not demonstrate the CVS.

One hundred fourteen of 391 assessed WM lesions were T1 black holes. Of these, $106 \mathrm{~T} 1$ black hole lesions (93\%) were CVS+. Conversely, 106 of 292 CVS + WM lesions (36\%) were T1 black holes.

The median proportion of CVS + WM lesions per participant was $87 \%$ (range, $29 \%-100 \%$ ). When we applied the $40 \%$ rule, ${ }^{8} 18$ (90\%) had $\geq 40 \%$ CVS + WM lesions (range: 55\%-100\%) and 2 (10\%) had $<40 \%$ CVS + WM lesions (29\% and 31\%) (Figs 1 and 2). Of note, the 2 individuals who did not meet the $40 \%$ rule did not have any visible infratentorial lesions or spinal cord lesions. These 2 individuals underwent initial brain MR imaging for work-up of pituitary adenoma and headache, which allowed identification of the WM lesions. When the WM lesions in these 2 individuals were scrutinized retrospectively, most were small $(<3$ $\mathrm{mm}$ ), punctate, rarely confluent, and located predominantly in the anterior subcortical and deep WM. Furthermore, these indi- viduals did not have WM lesions with T1 black holes or WM lesions involving the corpus callosum.

Nineteen individuals with RIS (95\%) fulfilled the rule of 6 criteria. ${ }^{10}$ The single individual who did not meet the rule of 6 also failed to meet the $40 \%$ rule (CVS+ WM lesions, 31\%). There was 1 participant who fulfilled the rule of 6 but failed to meet $40 \%$ rule (CVS+ WM lesions $=29 \%)($ Table 3$)$.

All the infratentorial lesions evaluated were CVS + $(13 / 13,100 \%)$. Ninetythree percent $(56 / 60)$ of periventricular WM lesions were CVS+, 78\% (70/90) of cortical/juxtacortical WM lesions were CVS+, and 67\% (153/228) of subcortical/deep WM lesions were CVS+ (Fig 3).

\section{Correlations between the Proportion of CVS + WM Lesions and Clinical/MR Imaging Variables}

Univariable regression analyses showed that the number of cervical spinal cord lesions was associated with a higher proportion of CVS + WM lesions $(P=.04)$. In multivariable regression models, including age, sex, number of brain WM lesions, cervical spinal cord lesions, and infratentorial lesions, age and the number of cervical spinal cord lesions showed a significant relationship with the proportion of CVS + WM lesions ( $P=.01$ and .002 , respectively), and the number of infratentorial lesions showed a trend toward a significant relationship $(P=.06)$. On the other hand, the total number of baseline WM lesions did not show a significant association with the proportion of CVS+ WM lesions $(P=.29)$ (Table 4).

\section{DISCUSSION}

In this study, we found that nearly all participants with RIS in our cohort had a high proportion of CVS + WM lesions, similar to that previously been reported in MS..$^{3,8,13-23,35}$ Furthermore, a large proportion of our RIS cohort (18/20, 90\%) met the $40 \%$ rule (Fig 1 and Table 3 ), ${ }^{8}$ which has previously been proposed to distinguish WM lesions observed in MS from other WM disorders. ${ }^{8}$

This is the first study assessing those with the CVS in RIS, a valuable population to evaluate because RIS is currently the earliest detectable stage of MS. Historically, asymptomatic inflammatory demyelinating lesions have been identified in postmortem series, with the estimated prevalence ranging from $0.08 \%$ to $0.2 \% \cdot{ }^{36-38}$ However, the true prevalence and natural history of RIS remain unknown, making this entity challenging to manage from a clinical perspective. The largest retrospective study to date suggests that $34 \%$ of individuals with RIS develop clinical MS during a follow-up of $\sim 5$ years. ${ }^{25}$

This study suggests that most MR imaging-visible lesions in RIS are consistent with perivenous demyelination, corroborating the notion that most of these individuals have typical MS pathol- 
ogy and are at risk of converting to MS. Identifying large proportions of CVS + WM lesions may have significant clinical implications for monitoring these participants and potentially initiating disease-modifying treatment. Prospective follow-up of this cohort will test the hypothesis that the proportion of CVS+ WM lesions in RIS has (either positive or negative) prognostic value. Furthermore, we also found T1 black holes in most subjects, and most T1 black holes demonstrated the CVS, suggesting that the observed WM lesions include destructive axonal pathology. It will also be of interest to evaluate the prognostic value of T1 black holes in RIS prospectively.

Current diagnostic criteria for MS emphasize lesion location as a helpful factor in the differential diagnosis of clinically isolated

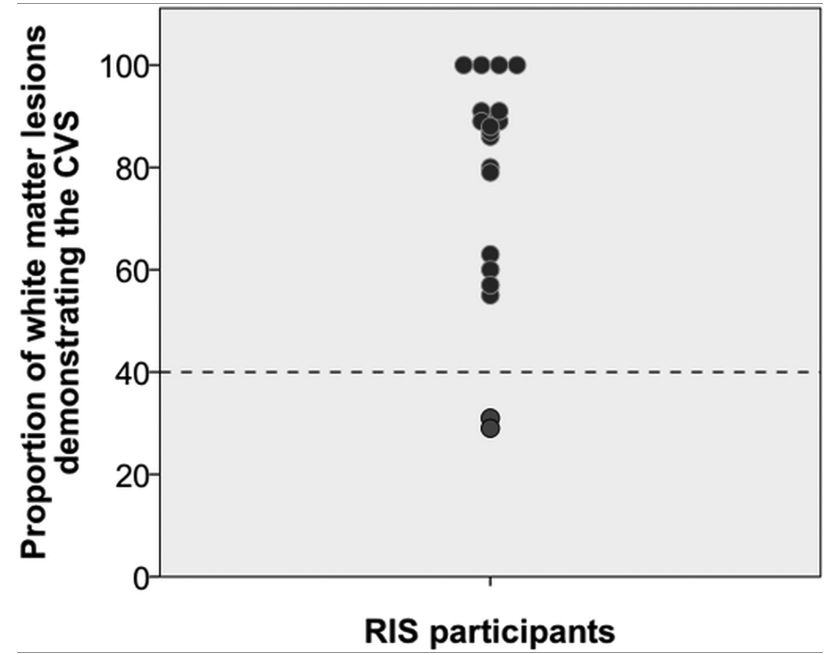

FIG 1. Proportion of white matter lesions demonstrating the central vein sign per case and the $40 \%$ rule. Eighteen patients with RIS (90\%) met the $40 \%$ rule, whereas 2 did not.
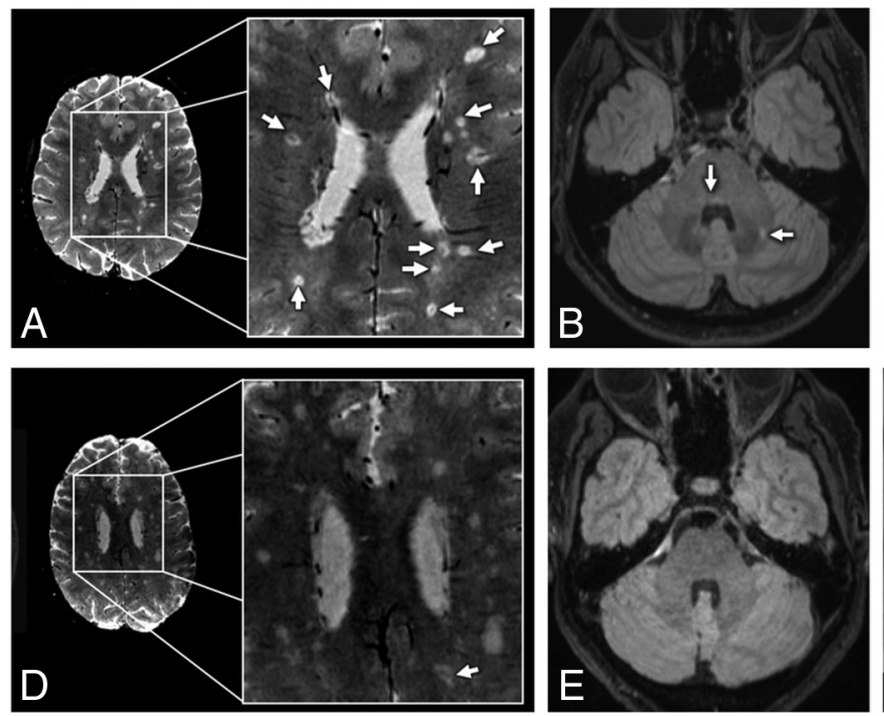

FIG 2. White matter lesions with evident central vein signs in 2 different radiologically isolated syndrome cases, as seen on axial reconstructed 3D-T2*-weighted segmented echo-planar imaging sequences and 3D-T2-weighted FLAIR sequences of the brain, and sagittal T1-weighted phasesensitive inversion recovery of the cervical spinal cord. A 50 -year-old woman in whom most $(90 \%)$ of the white matter lesions demonstrate the central vein sign ( $A$, arrows). This individual also has evident infratentorial ( $B$, arrows) and cervical spinal cord lesions ( $C$, arrow). A 53-year-old woman with a small proportion $(29 \%)$ of white matter lesions demonstrating the central vein sign $(D$, arrow). This individual did not have any infratentorial $(E)$ or cervical spinal cord lesions $(F)$.
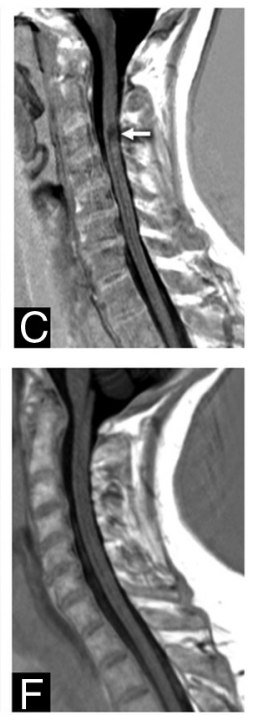

syndrome. When the WM lesions in our participants with RIS were classified by location, we found that the posterior fossa had the highest frequency of CVS + lesions, followed by periventricular and cortical/juxtacortical locations. On the other hand, subcortical and deep WM lesions had the lowest frequency of CVS+ lesions (Fig 3). Previous studies that assessed only supratentorial lesions ${ }^{8,35}$ found that the periventricular location had a high prevalence of CVS + lesions. Our observation of a very high proportion of CVS + lesions in the infratentorial region is of interest and is likely because lesions in the infratentorial region are not commonly seen with microangiopathy or migraines, common mimics of MS on MR imaging scans. The high prevalence of infratentorial

in patients in whom the diagnosis of MS is a possibility. ble 4). Given that previous studies have shown that the presence of a spinal cord lesion is one of the strongest predictors of RIS converting to MS, ${ }^{25,26,39}$ this finding raises the possibility that the proportion of CVS + WM lesions may similarly be a predictor of RIS conversion to MS. In addition, the number of infratentorial lesions in participants with RIS also showed a trend toward correlating with the proportion of CVS+ WM lesions, which, tosions in this region for the CVS may be of value from a prognostic standpoint. Given the cross-sectional nature of this study, however, these observations simply raise plausible hypotheses. Prospective follow-up of this cohort will be important to test these hypotheses before any definitive conclusions can be drawn.

A small proportion $(2 / 20,10 \%)$ of our RIS cohort had a low proportion of CVS+ WM lesions and failed to meet the $40 \%$ rule (Fig 1 and Table 3). ${ }^{8}$ Notably, both of these individuals had no observable infratentorial or cervical spinal cord lesions identified on MR imaging (Fig 2D-F). Taken together, these findings suggest that the WM lesions observed in these patients lack perivenous demyelination and that there is therefore a lower risk of developing clinical MS. Although these individuals were considered to have RIS based on the applied consensus criteria, in retrospect, the imaging pattern was more "nonspecific": Few lesions had the typical ovoid configuration of MS lesions. This finding highlights an important caveat of current RIS criteria, that lesions be typical of MS in the eyes of an expert observer. The determination of "typical" is necessarily subjective and explains why RIS cohorts may be heterogeneous. ${ }^{40,41}$ In other words, it is not surprising, given current criteria, that some individuals diagnosed with RIS 
Table 3: Comparison of CVS criteria for MS diagnosis in the RIS cohort

\begin{tabular}{lcc}
\hline \multirow{2}{*}{\begin{tabular}{l} 
Us Diagnostic Criteria \\
\cline { 2 - 3 } Using the CVS
\end{tabular}} & \multicolumn{2}{c}{ No. of RIS Participants } \\
\hline $40 \%$ rule & $18(90 \%)$ & Negative for CVS \\
Rule of 6 & $19(95 \%)$ & $2(10 \%)$ \\
\hline
\end{tabular}
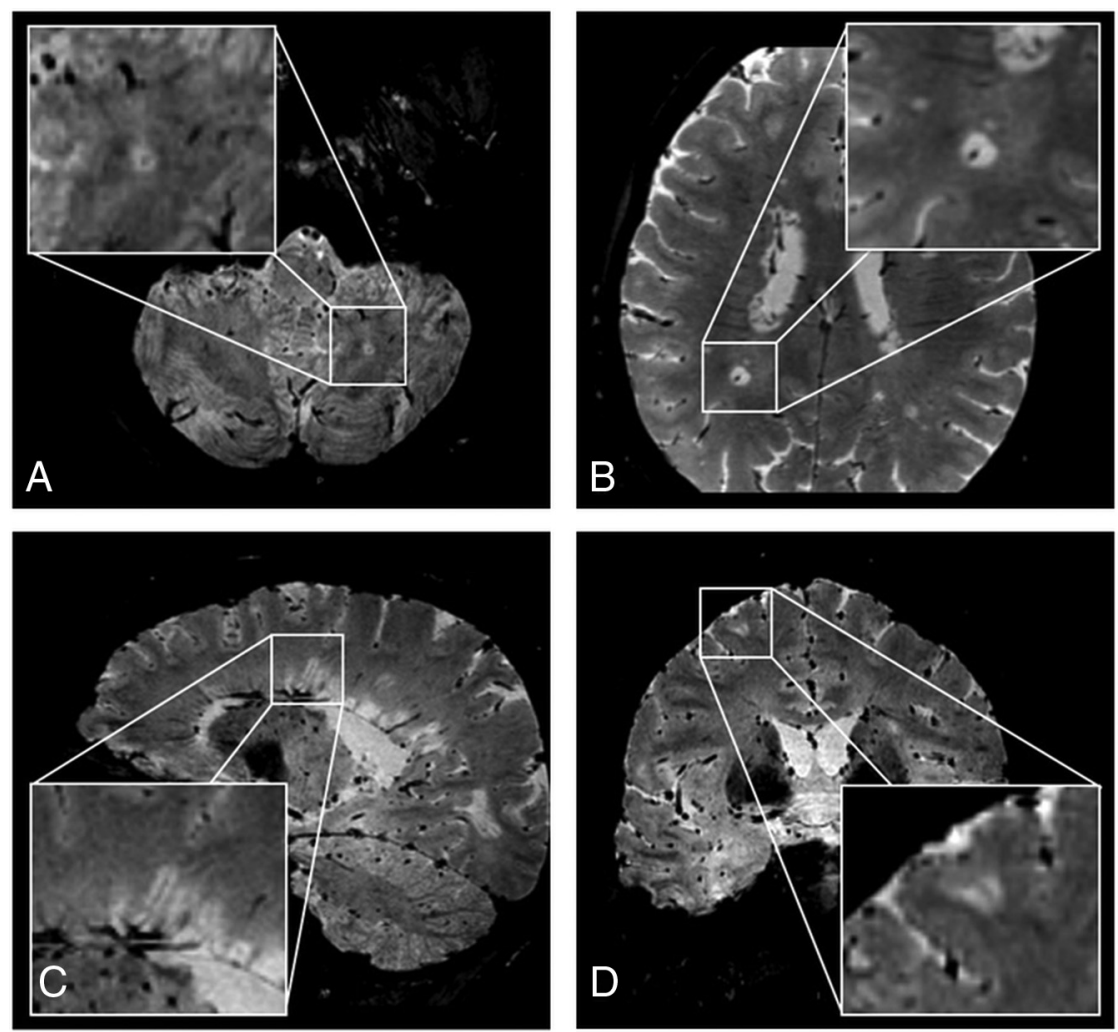

FIG 3. White matter lesions demonstrating the central vein sign in different brain regions, using 3D-T2*-weighted echo-planar imaging. A, Infratentorial. B, Deep white matter. C, Periventricular. $D$, juxtacortical.

Table 4: Relationships between the proportion of white matter lesions demonstrating the central vein sign and demographic and MRI variables in cases of RIS

\begin{tabular}{lcc}
\hline \multicolumn{1}{c}{ Variables } & $\begin{array}{c}\text { Univariable } \\
\text { Regression }(\boldsymbol{P} \text { Value })\end{array}$ & $\begin{array}{c}\text { Multivariable } \\
\text { Regression }(\boldsymbol{P} \text { Value) }\end{array}$ \\
\hline Age & .27 & .01 \\
Sex & .30 & .12 \\
Total No. of brain lesions & .33 & .29 \\
No. of infratentorial lesions & .21 & .06 \\
No. of cervical spinal cord lesions & .04 & .002 \\
\hline
\end{tabular}

distinguished MS from non-MS diseases, respectively. Because the lowest proportion of these 18 participants with RIS with $\geq 40 \%$ CVS + WM lesions was $55 \%$, applying either the $45 \%$ or $50 \%$ rule led to the same observation, highlighting that most participants with RIS had high CVS + WM lesions similar to those in MS.

When we applied the rule of $6,{ }^{10}$ the more simplified method to assess the CVS to our cohort, similar results were observed with most RIS participants, with the exception of 1 case. Only 1 case did not fulfill the rule of 6 , and this case similarly failed to meet the $40 \%$ rule. However, there was 1 case that failed to meet the $40 \%$ rule but met the rule of 6 . On further scrutiny, this participant had 16 CVS+ WM lesions of the $61 \mathrm{WM}$ lesions $(29 \%)$. By way of comparison, the individual who did not meet both sets of criteria had 5 CVS + WM lesions of a total $16 \mathrm{WM}$ lesions $(31 \%) .{ }^{10}$ These observations suggest that although there is general agreement between the 2 sets of criteria, the $40 \%$ rule may be more specific to individuals at risk of developing MS and the rule of 6 may need to be applied with caution in individuals with a large number of WM lesions. In line with this observation, another recent study in MS has shown that the rule of 6 has lower specificity for MS. ${ }^{21}$ Although applying the rule of 6 has the benefit of efficiency and simplicity over the $40 \%$ rule, in cases with high WM lesion loads, applying the $40 \%$ rule may prevent falsepositive conclusions. Given the practical difficulty of computing the CVS proportion in such cases, future directions may include the development of automated CVS detection algorithms ${ }^{42}$ that can be rapidly applied in clinical settings. A re-

may, in fact, have WM lesions related to other entities, such as migraines, microangiopathy related to vascular risk factors, or even other inflammatory disorders of the central nervous system. Recent studies that have evaluated the CVS in other inflammatory disorders, including neuromyelitis optica ${ }^{23}$ and central nervous system inflammatory vasculopathies, ${ }^{22}$ have shown a significantly higher proportion of CVS + WM lesions in MS. Thus, the availability of an imaging sign that is pathologically specific for MStype lesions, such as the CVS, should improve the diagnosis of RIS and allow appropriate identification of individuals who are truly at risk of developing MS, rather than other disorders.

The other studies have proposed other cutoff thresholds, including $45 \%,{ }^{10,21} 50 \%,{ }^{22}$ and $54 \%,{ }^{23}$ of which the proportion of CVS + WM lesions $\geq 45 \%$, $\geq 50 \%$, and $\geq 54 \%$ can be used to cent study reported a promising automated CVS-detection program that was able to distinguish MS from non-MS disease; however, the sensitivity and specificity of this program were lower than those in manually obtained CVS proportions, suggesting that refinements need to be made before such programs can be practically used in clinical settings.

This study has a number of limitations. First, the sample size was relatively small because RIS is a rare entity. Second, a significant proportion of WM lesions were excluded from CVS analysis in our RIS cohort (61\%), which is in accordance with current CVS identification criteria. ${ }^{11}$ In addition, due to the potential risks of gadolinium (ie, gadolinium retention and nephrogenic systemic fibrosis), our MR imaging protocol did not include a gadoliniumbased contrast agent, which has been demonstrated to increase 
visualization of central veins within small WM lesions on the 3DT2* EPI sequence due to its paramagnetic properties. ${ }^{9,11,12} \mathrm{Nev}-$ ertheless, the observed prevalence of CVS in our RIS individuals was high, and in light of our detailed findings in the 2 individuals with $<40 \%$ CVS + lesions, the administration of gadolinium would not likely have changed our conclusions. Furthermore, we were not able to compare the CVS in RIS with that in a healthy control group or with a group of patients with WM disorders other than MS; this comparison would have been of interest. However, a number of prior studies using similar methodologies have clearly demonstrated that the CVS is rarely found in healthy controls ${ }^{9,23,35,43}$ and to a much lower proportion in other WM disorders. ${ }^{3,8,11,15,21-23}$ Because this study was performed using sequences identical to those in recent studies and the CVS was evaluated by experienced investigators who coauthored prior studies, $3,9,11,12,15,21,22,42,43$ the lack of a control group would probably not have changed our conclusions. Finally, due to the cross-sectional nature of this study, we were unable to evaluate the prognostic value of the CVS in RIS. However, prospective follow-up of this cohort is planned, which will be informative and may eventually help guide clinical management.

\section{CONCLUSIONS}

In this study, we found that most individuals with RIS based on current diagnostic criteria have a high proportion of lesions demonstrating the CVS, similar to that seen in clinical MS. This finding suggests that most patients with RIS have perivenous demyelinating pathology typical of MS. Moreover, we found significant correlations between the proportion of CVS + WM lesions and spinal cord lesions in RIS, suggesting, by inference, that the CVS may have prognostic value in RIS. This raises the possibility that the CVS may be useful to distinguish patients with RIS at high risk of developing clinical MS from those with truly nonspecific WM lesions and therefore at minimal risk of developing clinical symptoms. Prospective follow-up of this cohort is planned, which will enable a better understanding of the diagnostic and predictive value of the CVS in RIS and of the natural history of RIS.

Disclosures: Suradech Suthiphosuwan-UNRELATED: Grants/Grants Pending: Sanofi Genzyme, Comments: I received fellowship educational support from an unrestricted educational grant. Xavier Montalban-UNRELATED: X.M. Consultancy: Biogen, Celgene, Sanofi Genzyme, Merck, Novartis, Roche and Teva Pharmaceutical. Daniel S. Reich—UNRELATED: Expert Testimony: Leventhal \& Puga, P.C. Jiwon OhRELATED: Grant: MS Society of Canada and Love/Barford Endowment*; UNRELATED: Consultancy: Biogen Idec, Roche, EMD Serono, Sanofi Genzyme, Celgene; Grants: Brain Canada, MS Society of Canada, Biogen Idec, Roche.* *Money paid to the institution.

\section{REFERENCES}

1. Thompson AJ, Baranzini SE, Geurts J, et al. Multiple sclerosis. Lancet 2018;391:1622-36 CrossRef Medline

2. Adams CW, Abdulla YH, Torres EM, et al. Periventricular lesions in multiple sclerosis: their perivenous origin and relationship to granular ependymitis. Neuropathol Appl Neurobiol 1987;13:141-52 CrossRef Medline

3. Solomon AJ, Schindler MK, Howard DB, et al. "Central vessel sign" on 3T FLAIR ${ }^{\star}$ MRI for the differentiation of multiple sclerosis from migraine. Ann Clin Transl Neurol 2016;3:82-87 CrossRef Medline

4. Polman CH, Reingold SC, Edan G, et al. Diagnostic criteria for multiple sclerosis: 2005 revisions to the "McDonald Criteria." Ann Neurol 2005;58:840-46 CrossRef Medline

5. Polman CH, Reingold SC, Banwell B, et al. Diagnostic criteria for multiple sclerosis: 2010 revisions to the McDonald criteria. Ann Neurol 2011;69:292-302 CrossRef Medline

6. Thompson AJ, Banwell BL, Barkhof F, et al. Diagnosis of multiple sclerosis: 2017 revisions of the McDonald criteria. Lancet Neurol 2018;17:162-73 CrossRef Medline

7. Tallantyre EC, Brookes MJ, Dixon JE, et al. Demonstrating the perivascular distribution of MS lesions in vivo with 7-Tesla MRI. Neurology 2008;70:2076-78 CrossRef Medline

8. Tallantyre EC, Dixon JE, Donaldson I, et al. Ultra-high-field imaging distinguishes MS lesions from asymptomatic white matter lesions. Neurology 2011;76:534-39 CrossRef Medline

9. Sati P, Thomasson DM, Li N, et al. Rapid, high-resolution, wholebrain, susceptibility-based MRI of multiple sclerosis. Mult Scler 2014;20:1464-70 CrossRef Medline

10. Mistry N, Abdel-Fahim R, Samaraweera A, et al. Imaging central veins in brain lesions with $3-\mathrm{T}$ T2*-weighted magnetic resonance imaging differentiates multiple sclerosis from microangiopathic brain lesions. Mult Scler 2016;22:1289-96 CrossRef Medline

11. Sati $\mathrm{P}, \mathrm{Oh}$ J, Constable RT, et al. The central vein sign and its clinical evaluation for the diagnosis of multiple sclerosis: a consensus statement from the North American Imaging in Multiple Sclerosis Cooperative. Nat Rev Neurol 2016;12:714-22 CrossRef Medline

12. Sati P, George IC, Shea CD, et al. FLAIR ${ }^{\star}$ : a combined MR contrast technique for visualizing white matter lesions and parenchymal veins. Radiology 2012;265:926-32 CrossRef Medline

13. Kau T, Taschwer M, Deutschmann H, et al. The "central vein sign": is there a place for susceptibility weighted imaging in possible multiple sclerosis? Eur Radiol 2013;23:1956-62 CrossRef Medline

14. Samaraweera AP, Clarke MA, Whitehead A, et al. The central vein sign in multiple sclerosis lesions is present irrespective of the $\mathrm{T} 2^{*}$ sequence at 3 T. J Neuroimaging 2017;27:114-21 CrossRef Medline

15. Solomon AJ, Watts R, Ontaneda D, et al. Diagnostic performance of central vein sign for multiple sclerosis with a simplified three-lesion algorithm. Mult Scler 2018;24:750-57 CrossRef Medline

16. Sparacia G, Agnello F, Gambino A, et al. Multiple sclerosis: high prevalence of the 'central vein' sign in white matter lesions on susceptibilityweighted images. Neuroradiol J 2018;31:356-61 CrossRef Medline

17. Miller AE, Calabresi PA. Central vein sign in multiple sclerosis: ready for front and center? Neurology 2018;90:631-32 CrossRef Medline

18. Inglese $\mathrm{M}$, Fleysher L, Oesingmann $\mathrm{N}$, et al. Clinical applications of ultra-high field magnetic resonance imaging in multiple sclerosis. Expert Rev Neurother 2018;18:221-30 CrossRef Medline

19. Hosseini Z, Matusinec J, Rudko DA, et al. Morphology-specific discrimination between MS white matter lesions and benign white matter hyperintensities using ultra-high-field MRI. AJNR Am J Neuroradiol 2018;39:1473-79 CrossRef Medline

20. Lamot U, Avsenik J, Sega S, et al. Presence of central veins and susceptibility weighted imaging for evaluating lesions in multiple sclerosis and leukoaraiosis. Mult Scler Relat Disord 2017;13:67-72 CrossRef Medline

21. Campion T, Smith RJP, Altmann DR, et al. FLAIR* to visualize veins in white matter lesions: a new tool for the diagnosis of multiple sclerosis? Eur Radiol 2017;27:4257-63 CrossRef Medline

22. Maggi P, Absinta M, Grammatico M, et al. Central vein sign differentiates multiple sclerosis from central nervous system inflammatory vasculopathies. Ann Neurol 2018;83:283-94 CrossRef Medline

23. Cortese R, Magnollay L, Tur C, et al. Value of the central vein sign at 3 to differentiate MS from seropositive NMOSD. Neurology 2018; 90:e1183-90 CrossRef Medline

24. Okuda DT, Mowry EM, Beheshtian A, et al. Incidental MRI anomalies suggestive of multiple sclerosis: the radiologically isolated syndrome. Neurology 2009;72:800-05 CrossRef Medline

25. Okuda DT, Siva A, Kantarci O, et al; Radiologically Isolated Syndrome Consortium (RISC), Club Francophone de la Sclérose en Plaques (CFSEP). Radiologically isolated syndrome: 5-year risk for an initial clinical event. PLoS One 2014;9:e90509 CrossRef Medline 26. Okuda DT, Mowry EM, Cree BA, et al. Asymptomatic spinal cord 
lesions predict disease progression in radiologically isolated syndrome. Neurology 2011;76:686-92 CrossRef Medline

27. Kantarci OH, Lebrun C, Siva A, et al. Primary progressive multiple sclerosis evolving from radiologically isolated syndrome. Ann Neurol 2016;79:288-94 CrossRef Medline

28. Barkhof F, Filippi M, Miller DH, et al. Comparison of MRI criteria at first presentation to predict conversion to clinically definite multiple sclerosis. Brain 1997;120(Pt 11):2059-69 CrossRef Medline

29. Filippi M, Rocca MA, Ciccarelli O, et al; MAGNIMS Study Group. MRI criteria for the diagnosis of multiple sclerosis: MAGNIMS consensus guidelines. Lancet Neurol 2016;15:292-303 CrossRef Medline

30. De Stefano N, Giorgio A, Tintoré M, et al. Radiologically isolated syndrome or subclinical multiple sclerosis: MAGNIMS consensus recommendations. Mult Scler 2018;24:214-21 CrossRef Medline

31. Forslin Y, Granberg T, Jumah AA, et al. Incidence of radiologically isolated syndrome: a population-based study. AJNR Am J Neuroradiol 2016;37:1017-22 CrossRef Medline

32. Alcaide-Leon P, Pauranik A, Alshafai L, et al. Comparison of sagittal FSE T2, STIR, and T1-weighted phase-sensitive inversion recovery in the detection of spinal cord lesions in MS at 3T. AJNR Am J Neuroradiol 2016;37:970-75 CrossRef Medline

33. Huo Y, Carass A, Resnick SM, et al. Combining multi-atlas segmentation with brain surface estimation. Proc SPIE Int Soc Opt Eng 2016; 9784 Medline

34. Nair G, Absinta M, Reich DS. Optimized T1-MPRAGE sequence for better visualization of spinal cord multiple sclerosis lesions at $3 \mathrm{~T}$. AJNR Am J Neuroradiol 2013;34:2215-22 CrossRef Medline

35. Kilsdonk ID, Lopez-Soriano A, Kuijer JP, et al. Morphological fea- tures of MS lesions on FLAIR $^{*}$ at $7 \mathrm{~T}$ and their relation to patient characteristics. J Neurol 2014;261:1356-64 CrossRef Medline

36. Engell T. A clinical patho-anatomical study of clinically silent multiple sclerosis. Acta Neurol Scand 1989;79:428-30 CrossRef Medline

37. Gilbert JJ, Sadler M. Unsuspected multiple sclerosis. Arch Neurol 1983;40:533-36 CrossRef Medline

38. Castaigne P, Lhermitte F, Escourolle R, et al. Asymptomatic multiple sclerosis: 3 cases (author's transl) [in French]. Rev Neurol (Paris) 1981;137:729-39 Medline

39. Granberg T, Martola J, Kristoffersen-Wiberg M, et al. Radiologically isolated syndrome: incidental magnetic resonance imaging findings suggestive of multiple sclerosis-a systematic review. Mult Scler 2013;19:271-80 CrossRef Medline

40. Labiano-Fontcuberta A, Benito-Leon J. Radiologically isolated syndrome: an update on a rare entity. Mult Scler 2016;22:1514-21 CrossRef Medline

41. Liu S, Kullnat J, Bourdette D, et al. Prevalence of brain magnetic resonance imaging meeting Barkhof and McDonald criteria for dissemination in space among headache patients. Mult Scler 2013;19: 1101-05 CrossRef Medline

42. Dworkin JD, Sati P, Solomon A, et al. Automated integration of multimodal MRI for the probabilistic detection of the central vein sign in white matter lesions. AJNR Am J Neuroradiol 2018;39: 1806-13 CrossRef Medline

43. Gaitń MI, de Alwis MP, Sati P, et al. Multiple sclerosis shrinks intralesional, and enlarges extralesional, brain parenchymal veins. Neurology 2013;80:145-51 CrossRef Medline 